\title{
Germanica
}

GERMANICA

$53 \mid 2013$

Littérature et cinéma dans l'espace germanophone contemporain : jeux intermédiaux, modes de transfert, adaptations

\section{Les yeux des autres}

Entretien entre Alexander Kluge et Vincent Pauval

Traducteur : Vincent PAUVAL

\section{OpenEdition}

\section{Journals}

Édition électronique

URL : http://journals.openedition.org/germanica/2297

DOI : 10.4000/germanica.2297

ISSN : 2107-0784

Éditeur

Université de Lille

Édition imprimée

Date de publication : 30 décembre 2013

Pagination : 251-272

ISBN : 9782913857322

ISSN : 0984-2632

Référence électronique

«Les yeux des autres », Germanica [En ligne], 53 | 2013, mis en ligne le 27 janvier 2015, consulté le 06 octobre 2020. URL : http://journals.openedition.org/germanica/2297 ; DOI : https://doi.org/10.4000/ germanica.2297

(c) Tous droits réservés 


\section{Les yeux des autres}

\section{Entretien entre Alexander Kluge et Vincent Pauval ${ }^{1}$}

Dans cet entretien enregistré le samedi 14 septembre 2013, le grand écrivain et cinéaste Alexander Kluge (*1932) revient sur l'évolution spectaculairement prolifique de son æuvre depuis la fin des années 1980 et depuis le tournant du millénaire notamment. Moins connue en France, cette période-là de sa production artistique a vu néanmoins la parution d'une somme littéraire aussi surprenante qu'impressionnante, dont la traduction intégrale en langue française est en cours actuellement ${ }^{2}$. Quant au cinéma de Kluge, il a fait l'objet d'une rétrospective complète à la Cinémathèque française (Bercy) du 24 avril au 3 juin 2013, un an après avoir été à l'honneur au Festival international du court-métrage de Clermont-Ferrand durant l'hiver 2012. À cette même occasion, le

1. - ATER, Université Blaise Pascal de Clermont-Ferrand, spécialiste et traducteur d'Alexander Kluge.

2. - Le premier ouvrage de sa plume récemment paru en langue française est celui que l'auteur a conçu avec le peintre, photographe et plasticien Gerhard Richter : Décembre, Zurich-Biel, Diaphanes, 2012 (traduit de l'allemand par Hilda Inderwildi et Vincent Pauval). Voir aussi le dossier d'environ quarante pages paru dans la revue de création Fario, n 12 , Paris, 2013 ; ce dossier comprend d'autres récits de Kluge écrits pour Gerhard Richter et émaillés de reproductions d'œuvres de ce dernier, une présentation substantielle de la part des traducteurs Hilda Inderwildi et Vincent Pauval, ainsi qu'une interview de l'écrivain réalisée par Vincent Pauval. Sont également parues 16 histoires pour Anselm Kiefer (traduites de l'allemand par Hilda Inderwildi et Vincent Pauval) dans le catalogue de l'exposition d'Anselm Kiefer donnée à la Galerie Thaddaeus Ropac du 14 octobre 2012 au 27 janvier 2013 : Anselm Kiefer. Die Ungeborenen, Galerie Thaddaeus Ropac, Paris/Pantin, 2013. Enfin, à compter de l'automne 2014, l'intégralité de l'œuvre littéraire d'Alexander Kluge paraîtra en plusieurs volumes sous la direction de Vincent Pauval aux éditions P.O.L sous le titre général de Chronique des sentiments.

GERMANICA, 2013, LIII, p. 251 à 272. 
colloque interdisciplinaire "Alexander Kluge et la France - Pour une levée en masse de la narration ${ }^{3} »$ a été organisé par Éric Lysфe et Vincent Pauval à la Maison des Sciences de l'Homme de Clermont-Ferrand, afin de fournir au lectorat français un premier aperçu global des caractères essentiels à l'ceuvre immense de cet auteur relativement peu étudié jusque-là en France. L'entretien qui suit a vocation de dresser un état des lieux de la réflexion klugienne autour des moyens du cinéma et de la littérature, de leurs rapports ainsi que de leurs potentialités dans le contexte historico-médiatique contemporain.

\section{Vincent Pauval : Comment avez-vous vécu l'année 1989 et le début du grand « tournant » pour l'Allemagne entamé alors?}

Alexander Kluge : Plutôt passivement. Je suis originaire d'Halberstadt, une ville qui se situe sur le territoire de la RDA et dans laquelle mon père a vécu jusqu'en 1979. Pour ma part, j'avais quitté cet endroit dès 1946. Par la suite, je suis devenu un citoyen de la République Fédérale d'Allemagne à part entière. Au fond, l'idée d'une «Allemagne réunifiée », à laquelle je n'étais plus habitué, me paraissait improbable, au même titre que Berlin comme capitale. Je suis donc un patriote de la RFA, mais de l'ancienne RFA avec pour capitale Bonn, plus ancrée à l'ouest.

V.P. : Au moment de la parution de votre ouvrage Chronique des sentiments ${ }^{4}$ en 2000, vous avez déclaré vous être remis à écrire de plus belle après la réunification allemande. Comment vous l'expliquez-vous?

A.K. : En fait, ceci était moins lié à la réunification qu'à ce que la période ayant suivi l'année 1991 et la fin de la Guerre froide m'a semblée marquer un nouveau commencement. Pour mes enfants, je voyais naître une nouvelle ère augustéenne, pour laquelle il valait la peine de conserver les expériences antérieures. Il reste tout de même l'expérience amère de la Seconde Guerre mondiale, l'expérience de l'entre-deux guerres, et l'impression de la Guerre froide, avec toute l'inquiétude qu'elle m'a inspiré : «Inquiétance du temps 5 », voilà

3. - Les actes de ce colloque seront publiés très prochainement aux Presses Universitaires Blaise Pascal (PUBP) de Clermont-Ferrand.

4. - Alexander Kluge, Chronik der Gefühle, Francfort-sur-le-Main, Suhrkamp, 2000. Chef-d'œuvre de Kluge, cet ouvrage d'environ 2000 pages compile l'ensemble des textes narratifs parus en volumes individuels jusqu'à la fin des années 1970, augmenté de centaines d' « histoires » que l'auteur avait rédigées depuis, sans néanmoins publier de nouveau recueil pendant plus de vingt ans.

5. - Nous citons la traduction qu'a donnée Herbert Holl du titre d'un important recueil d'Alexander Kluge : Neue Geschichten. Hefte 1 - 18>Unheimlichkeit der Zeit<, Francfort-sur-le-Main, Suhrkamp, 1977. Le recueil figure dans le second volume de 
ce qu'elle était pour moi. Et nous voici tout d'un coup soulagés de ce cauchemar. Cela tenait moins à la réunification allemande qu'à la perspective évidente d'une détente politique à l'échelle mondiale, perspective qu'à l'heure actuelle, en 2013, nous avons du reste déjà gâchée.

\section{V.P. : Il n'empêche que de nombreux auteurs ont vécu l'effondrement} du bloc de l'Est comme le déclin d'une utopie, le tarissement d'une source de motivation voire d'inspiration.

A.K. : Je n'ai jamais ressenti le bloc de l'Est comme une utopie. Comme Heiner Müller ou d'autres auteurs préoccupés par la question, le recul émotionnel que je prends me fait remonter jusqu'à la fin de la Première Guerre mondiale en 1917-1918. À cette époque la politique en général, jugée trop molle, subissait une remise en cause. Selon moi, le socialisme concerne avant tout la question de savoir pourquoi la classe productive parmi les hommes ne parvient pas à empêcher une telle catastrophe guerrière. Une politique et une classe politique incapables d'éviter un 1er août 1914 y laissent leur autorité. J'ai beaucoup réfléchi à cela, non pas l'enfant que j'étais, réfugié dans un abri antiaérien pendant les bombardements de 1945, mais bien pendant les années de 1977 à 1989, de l'automne allemand jusqu'à la réunification.

\section{V.P. : Après la parution du livre de Francis Fukuyama, on a voulu croire soudain que la fin de l'histoire ${ }^{6} s$ 'annonçait. Que pensez-vous de cette idée ?}

A.K. : Je parlerais plutôt d'un retour de l'histoire. Elle a fait défaut en 1914 pour finir en cauchemar, avec Auschwitz, et elle est de nouveau au rendez-vous en 1989, comme elle l'est encore aujourd'hui. J'ai été très étonné d'apprendre que les versements relatifs au Plan Young, qui réglementait le paiement des réparations suite au Traité de Versailles, n'ont été acquittés qu'en 1990. On mesure là toute l'étendue des chaînes causales de l'histoire.

\section{V.P. : En quoi l'histoire allemande vous intéressait-elle et en quoi vous intéresse-t-elle de nos jours, à l'heure de la mondialisation ?}

A.K. : L'histoire allemande a beaucoup perdu de son intérêt, mais elle demeure un laboratoire de toutes les erreurs que je puis imaginer au plan politique. C'est pourquoi j'ai le sentiment que le transfert aux

Chronik der Gefühle, à laquelle il tient lieu de chapitre 8.

6. - Cf. Francis Fukuyama, La Fin de l'histoire et le dernier homme, Paris, Flammarion / Champs, 1993. 
générations futures, le récit de l'expérience dans toute sa richesse, est primordial. L'histoire allemande jusque dans ses racines est un laboratoire du malheur. Et il faut raconter cette histoire malheureuse, afin qu'elle ne concerne plus seulement l'Allemagne, car l'expérience qu'elle renferme est universelle.

\section{V.P. : Peu après la chute du Mur, votre ami Heiner Müller déclarait que « l'Allemagne a constitué un sujet dramatique valable, jusqu'à la réunification $\gg 7$. De quelle manière commenteriez-vous cette affir- mation?}

A.K. : Je ne suis pas dramaturge. Par conséquent, je ne considère pas l'Allemagne qui a suivi la réunification ni celle qui l'a précédée sous l'angle de sa valeur dramatique. Du point de vue épique et poétique, dont la méthode m'est familière, je ne vois pas tellement de différence entre l'avant et l'après. Il y a toujours des sujets comme les 500 années de la Réforme par exemple, ou encore la Guerre des Paysans allemands, qui remonte à la même époque, et qui restent d'actualité même s'ils ne jouent aucun rôle dans le champ de la realpolitik. Les éléments du passé survivent à l'intérieur des individus. Ils forment des prismes et des cristaux, comme dirait Walter Benjamin. J'ignore s'il est possible de représenter cela sous forme de drame. Je sais en revanche que s'il n'est pas matière à créer des événements théâtraux, le sujet se prête à la recherche, c'est-à-dire au commentaire narratif.

\section{V.P. : Parmi vos modèles favoris, vous évoquez souvent Heiner Müller d'une seule traite avec Ovide, Tacite et Montaigne. Comment associez-vous ces noms?}

A.K. : Les Métamorphoses d'Ovide représentent pour moi la forme littéraire, voyez-vous. Elles enchaînent des variations qui forment un réseau. Sauf que mon approche de l'Antiquité passe toujours par quelqu'un que je perçois comme mon contemporain, à l'instar d'Ossip Mandelstam qui apprécie Ovide tout autant, au point d'avoir baptisé son œuvre tardive Tristia ${ }^{8}$ d'après un titre d'Ovide, ou encore de Heiner Müller, doué d'une compréhension très exacte de l'œuvre d'Ovide et avec qui je m'en suis beaucoup entretenu. C'est ainsi qu'Ovide devient mon contemporain. Il en va de même avec Montaigne qui, par ses commentaires, ses Essais, est à mes yeux le représentant d'une forme littéraire demeurée inaccomplie en littéra-

7. - Heiner Müller, Krieg ohne Schlacht - Leben in zwei Diktaturen - Eine Autobiographie, Cologne, Kiepenheuer \& Witsch, 1992, p. 267 : «Deutschland war ein gutes Material für Dramatik, bis zur Wiedervereinigung. »

8. - Cf. Ossip Mandelstam, Tristia et autres poèmes, Paris, Gallimard (coll. «Poésie »), 2005. 
ture. Je serais fier de pouvoir être identifiable comme son parent ou son successeur dans sa manière d'écrire.

\section{V.P. : Dans quelle mesure un poète de la RDA comme Heiner Müller pouvait-il influer sur votre conception de la littérature ? Qu'est-ce qui vous reliait?}

A.K. : Nous sommes apparentés poétiquement l'un à l'autre. Nous avons tous deux des origines slaves à travers une partie de nos ancêtres. Nous sommes capables chacun de parler comme on s'exprime aux endroits où nous sommes nés. Nous sommes voisins par nos tempéraments. Bien que les résultats poétiques soient très différents, nous puisons à la même source.

V.P. : De 1987 à 1995, vous avez mené de nombreux entretiens avec Heiner Müller dans le cadre de vos magazines télévisés 9 . En quoi ces programmes se distinguent-ils d'entretiens d'auteur conventionnels ?

A.K. : Ils diffèrent déjà en ce qu'ils ne suivent pas les règles dictées par la télévision. Car Heiner Müller ne répond pas à des questions typiques de ce média, mais à celles d'un auteur. Au fond, je considère comme une forme de littérature cette manière de conférer oralement tout en enregistrant. Cette forme est selon moi souvent plus libre que l'écrit. Chacun prête attention à l'autre. Dans bien des cas Heiner Müller ne répond d'ailleurs pas directement, mais une demi-heure plus tard, parlant d'autres sujets dans l'intervalle. Chacun se défait de l'armure de son moi. Cela correspond à ce que dit Heinrich von Kleist à propos de «l'élaboration progressive des idées par la parole » 10 .

V.P. : Mais la télévision saurait-elle passer pour une continuation de la littérature ou du cinéma à proprement parler ? La télévision ne tendrait-elle pas plutôt à écarter aussi bien l'un que l'autre?

A.K. : Disons que cela est hors de sa portée, puisque la littérature reste autonome par rapport à la télévision. D'une part, je n'ai jamais pu découvrir la moindre œuvre ou performance ni quelque forme littéraire que ce soit à la télévision. D'autre part, celle-ci reste un média dominant : lorsqu'arrive un événement inhabituel tel que les attentats du 11 Septembre 2001, je me tourne instinctivement vers ce média, un peu comme si de ma fenêtre je regardais la place du marché,

9. - L'intégralité de ces entretiens est disponible en ligne sur le site qui leur est consacré par l'Université de Brême et la Cornell University Library : http://muller-kluge. library.cornell.edu/de/index.php.

10. - Voir l'essai de Heinrich von Kleist, Über die allmähliche Verfertigung der Gedanken beim Reden, in : Sämtliche Erzählungen und andere Prosa, Stuttgart, Reclam, 2000, p. 340-346. 
comme on faisait jadis pour voir s'il s'y passe quelque chose. Un média dominant détient la confiance des gens qui s'attendent à ce que toute chose importante soit annoncée là en premier. Désormais, cela n'est plus du tout vrai, car il serait nettement devancé par Internet, mais cela reste une voie familière, comme le demeure aussi la place du marché même lorsqu'elle ne donne plus lieu à aucun marché ni à aucun attroupement. Il vaut la peine d'introduire au cœur d'un tel média quelques traces du meilleur dont on dispose, c'est-à-dire la musique et la littérature, sans le modifier ni l'adapter aux besoins télévisuels, mais à la manière d'un corps étranger, comparable à un bloc erratique, de ces rochers du Grand Nord que les glaciers ont charriés vers la plaine, un milieu qui n'est pas leur décor naturel. La poésie à la télévision ressemble un peu à cela, elle y devient possible dans les coins reculés, le plus souvent la nuit.

\section{V.P. : Et si les émissions ainsi produites débouchaient au contraire} sur de la littérature, quand par exemple lesdits entretiens sont publiés sans images, sous forme de livres ? Quelle signification ou quelle valeur accordez-vous à ce type de recueil ${ }^{11}$ ?

A.K. : Il s'agit-là de procès-verbaux. Mais il ne serait guère fortuit que les versions écrites de ces entretiens soient ensuite représentées par des comédiens, pourquoi pas de la Comédie Française. Cela rend assez bien, c'est particulier, certes, et laisse une impression assez étrange. $\mathrm{Au}$ fond, ces entretiens correspondent à une forme narrative ancestrale qui repose sur l'oralité. Cette dernière ne consiste pas en ce qu'un poète s'installe au milieu d'une salle pour y déclamer ses textes, mais à ce que des réponses aient lieu. L'idéal est la ronde. Au $\mathrm{XI}^{\mathrm{e}}$ siècle, une convention faisait qu'en Provence les gens se rassemblaient la nuit, chantaient des chansons, puis racontaient une histoire, chantaient d'autres chansons et se remettaient à raconter des histoires. C'est là une forme littéraire ancestrale, celle de la chantefable, et qui fut en même temps l'ancêtre de l'opéra.

V.P. : Dans quelle mesure le rôle que vous tenez ici est-il celui d'un auteur, sachant qu'au cours de ces entretiens votre personne se positionne le plus souvent en marge ?

11. - Ces entretiens ont été réunis et, pour une grande partie, publiés par Alexander Kluge en deux recueils : "Ich schulde der Welt einen Toten (Berlin, Rotbuch, 1995) et «Ich bin ein Landvermesser » (Berlin, Rotbuch, 1996). Les deux volumes sont respectivement parus en langue française sous les titres Esprit, pouvoir et castration (1997) et Profession arpenteur (2000) aux éditions Théâtrales (traduits de l'allemand pas Eleonora Rossi et Jean-Pierre Morel). 
A.K. : Mon rôle équivaut à celui d'un esprit invisible et bienveillant. Car ces entretiens procèdent suivant le mode animiste. Ce qui veut dire que les gens commencent à raconter des histoires lorsqu'ils partagent un enthousiasme. Lorsqu'ils sont dans la détresse et que celle-ci leur donne un moment de répit, ils se mettent aussi à raconter. Et quand ils en réchappent, ils remettent cela encore. Shéhérazade en constitue la base, une forme dont le principe est comparable à celui de la chantefable, en plus développé, l'Orient ayant eu plus de temps pour élaborer ce genre de choses. Mais il n'y a pas que la Shéhérazade du Bagdad des Contes des mille et une nuits, puisqu'il existe un recueil des musulmans occidentaux, issu de la grande culture de Cordoue en Espagne, qu'est celui des Cent et une nuits, une compilation apparentée à la précédente, et dont les histoires paraissent contées d'une façon encore plus rudimentaire, plus orale encore que dans les fameux Contes des mille et une nuits. Les Cent et une nuits sont un emblème de la littérature, un livre collectif, une compilation née de sources musulmanes occidentales de l'Espagne, par opposition au recueil persan.

\section{V.P. : L'auteur que vous êtes ne s'exprime-t-il pas davantage désor- mais comme écrivain?}

A.K. : Si vous le dites ainsi, je constate en effet que j'écris nettement plus depuis l'an 2000 à peu près, et que je progresse plus hardiment sur ce terrain-là où j'expérimente davantage qu'en d'autres médias. J'attribue cela à l'immense pression du réel émanant des médias, qui stimule et lance ses défis, mais qui exerce aussi une influence paralysante, m'incitant plus fortement à me réfugier dans l' " oasis » du livre. Je recherche donc intuitivement une caverne, une oasis, un cadre assez solide pour résister à cette entreprise monstrueuse qui détruit aussi des cadres. Le média en soi n'est pas en cause, mais ce qu'il implique en termes de forces participantes, ses usagers aux appétits voraces et cannibales.

V.P. : Il vous arrive aussi de désigner la production de dialogues
authentiques, dont vous donnez par ailleurs des variations fictives
dans votre ouvre littéraire, comme des œuvres «filmiques », et c'est
comme « films » que vos entretiens avec Heiner Müller ont été pré-
sentés à la Cinémathèque de Paris. Comment faut-il entendre cela?

A.K. : En regardant mes films, vous allez trouver en miniature le même type de dialogue, y compris parmi les choses que la caméra observe pendant que le texte fournit un commentaire. On voit à l'image par exemple une flaque d'eau et la surface de cette eau frémissant au vent. Cette image se suffit à elle-même. Et le commentaire indique: 
cette flaque possède tout son temps. Pendant ce temps, l'action se précipite vers un état de guerre. Voilà ma façon de faire, ma définition de ce qu'est un film. On peut la rapprocher du spectacle offert par deux individus qui se prêtent attention réciproquement et de manière prolongée. Il s'agit là d'une section de film au même titre que si je montais une scène d'amour. Pour celle-ci j'éviterais d'ailleurs tout autant de fournir des dialogues ou quelque indication que ce soit pour le jeu d'acteur. Au contraire, je bâtirais un cadre permettant aux deux acteurs de se sentir authentiques et de ressentir réellement de l'amour. Il suffit qu'ils se souviennent.

\section{V.P. : L'aspect de la fiction serait donc secondaire par rapport à l'authenticité ?}

A.K. : Le fait qu'une chose soit authentique ne dépend pas, en effet, de ce que celle-ci soit inventée de toutes pièces et soit le résultat d'un désir ou d'une transformation subjective, ou de sa dimension objective, c'est-à-dire tangible. Les termes comme « fictionnel » ou « véritable » correspondent à un ordre purement gestionnaire, à des rubriques. Cela dit, il n'est pas indifférent d'écrire par exemple des dialogues auxquels les acteurs donneront forme et prêteront voix, ou de les investir d'un rôle en permettant qu'ils improvisent. Mais les deux approches se valent, car les enjeux du cinéma et de la littérature ne sont pas de chercher une "Vérité » ni de distinguer le vrai du faux, mais résident dans les tensions, les équilibres, les courants, les champs de perception avec lesquels nous devons composer. Ainsi, je ne vois pas de distinction entre le fictionnel et le documentaire. Il est possible, en revanche, de vérifier l'immédiateté et l'authenticité d'une expérience, d'où la validité du principe d'authenticité. Sans cela un dialogue ne vaut rien.

V.P. : Votre récit Production filmique d'un texte ${ }^{12}$ illustre comment le dialogue d'un film peut devenir littérature. Vous y relatez la manière dont a été réalisée l'une des scènes les plus célèbres de votre premier long métrage Anita $G$., où l'héroïne se dispute avec sa logeuse...

A.K. : ... qui met Anita G. à la porte parce que celle-ci ne peut pas régler son loyer. Le dialogue engagé entre ces deux femmes, c'est-à-dire ma sœur dans le rôle d'Anita G. ${ }^{13}$ et la propriétaire aisée qu'est cette logeuse francfortoise, ces deux personnes l'inventent dans le feu de

12. - Voir le recueil d'Alexander Kluge, Geschichten vom Kino, Francfort-sur-leMain, Suhrkamp, 2007, p. 267-269.

13. - Alexandra Kluge, sœur d'Alexander, a assumé plusieurs premiers rôles dans les premiers longs métrages de son frère, dont celui de l'héroïne éponyme d'Anita $G$. (Abschied von Gestern, 1965). 
leur énervement comme j'aurais peine à le faire par l'écriture. En tant qu'auteur, je me contente donc de créer une situation qui permet aux personnages de s'énerver. Ceci n'est pas faisable artificiellement. Il se passe quelque chose entre les individus qu'à vrai dire le film est seul capable de retenir. Un écrivain serait en mesure de le réécrire, mais on ne saurait l'imiter ou l'inventer. En un tout autre lieu, ma sœur avait déjà une fois été mise à la porte de son appartement et, en tous cas, elle comprend ce que cela signifie que d'être mis à la rue. Sa partenaire, la logeuse, a eu affaire au fil des ans à une vingtaine de locataires malhonnêtes. Toute sa colère ainsi accumulée pénètre cette scène. Dans ce creuset d'alchimiste qu' on appelle une scène, quelque chose s'articule qui n'a aucune existence véritable à cet instant. Un tel moment scénique est aussi créateur et permet autant de concentration que le cerveau de l'auteur littéraire, sauf que cela se passe en dehors de sa tête, car en l'occurrence l'auteur ne peut fixer qu'un cadre.

\section{V.P. : Ce que vous décrivez-là correspond en fait à ce que Bakhtine a montré à partir de la poétique de Dostoïevski ${ }^{14}$ en théorisant la notion de dialogisme : des discours et des formulations antérieures à la situation sont actualisés et réactivés à travers elle.}

A.K. : Tout à fait ! Et dans ce film j'instaure des conditions qu'on ne trouve pas dans la vraie vie. Dans la vraie vie il n'y aurait pas d'équipe de tournage, pas plus qu'il y aurait d'autres scènes qui précèdent l'événement ou en découlent. Mais la concentration que je crée de la sorte donne à cette femme qui, d'habitude, ne se focalise pas sur l'intégralité de sa colère et qui ne se fâche qu'à un degré "normal », la capacité de faire entrer dans son jeu un concentré de colère, c'està-dire d'en donner la représentation. Ceci vaut également pour ma sœur, qui ne court pas le monde en rouspétant. D'une nature aimable, elle devient pourtant capable ici de s'emporter au point de répliquer presque avec hargne. Et voici comment une réplique appelle la suivante. Le ton monte progressivement entre les deux, puis se maintient à son niveau le plus élevé, avant que la scène soit ponctuée par la mise à la porte. C'est là une façon de générer de l'authenticité - peu importe qu'elle soit littéraire ou filmique - qui ne se fonde pas sur les conceptions d'usage ou sur un scénario, c'est-à-dire sur des textes composés dans un bureau, car il n'est pas possible de produire cela dans un atelier. La méthode est empruntée à la Nouvelle Vague.

V.P. : Mais ne serait-il pas justifié de nos jours d'affirmer que l'auteur réalisateur qui suivait le modèle de la Nouvelle vague française

14. - Cf. Mikhail M. Bakhtine, Problèmes de la poétique de Dostö̈evski, Lausanne, L'Âge d'Homme (coll. « Slavica »), 1970. 
durant les années 1960 est entre-temps devenu un producteur de films qui place le collectif au centre, faisant disparaître pour ainsi dire l'auteur Kluge en mettant la coopération au premier plan ?

A.K. : Vous avez raison de le souligner. Cette méthode, qui au fond ne date pas d'hier, vous la trouvez annoncée dans le cinéma révolutionnaire russe, par Poudovkine ${ }^{15}$ et Tretiakov ${ }^{16}$. Jean-Luc Godard ${ }^{17}$ lui aussi doit non seulement beaucoup au cinéma muet, mais également au cinéma russe.

V.P. : Votre récent film Nouvelles de l'antiquité idéologique ${ }^{18}$ constitue un exemple assez éloquent de l'application du principe de coopération. Peut-on parler, ne serait-ce qu'au plan formel, d'une continuité par rapport aux films collectifs, très engagés également, que vous avez produits durant les années 1970 et 1980 ?

A.K. : L'Allemagne en automne ${ }^{19}$ définit déjà le cadre formel, un cadre nouveau d'ailleurs, puisqu'il s'agit d'un film collectif né d'une incitation politique particulière. Nouvelles de l'antiquité idéologique en serait pour ainsi dire la continuation avec d'autres moyens, sachant qu'en l'occurrence je dispose d'un atout majeur, puisque j'ai la possibilité de travailler dans l'optique d'Eisenstein ${ }^{20}$. Car on peut voir un homme qu'on vénère autant que je vénère Eisenstein comme une lentille de caméra à travers laquelle on regarde, une caméra imaginaire avec laquelle il est possible d'enregistrer. C'est ce qu'on aura appris de notre collaboration autour des films collectifs des années 1970 et 1980, mais il est possible d'en étendre l'application, comme c'est ici le cas. Il n'est donc pas fortuit que Tom Tykwer ${ }^{21}$ ait apporté à

15. - Vsevolod Poudovkine (1893-1953).

16. - Sergueï Tretiakov (1892-1937).

17. - Jean-Luc Godard $(* 1930)$ reste une référence importante pour Kluge, qui se plaît à citer À bout de souffle (1960) comme l'un de ses films préférés. Il existe aussi un entretien mémorable de Kluge avec Godard («Blinde Liebe ») disponible en supplément sur le dvd n ${ }^{\circ} 15$ de l'édition complète de ses longs métrages: Sämtliche Kinofilme, Francfort-sur-le-Main, Zweitausendeins, 2007.

18. - Alexander Kluge, Nachrichten aus der ideologischen Antike. Marx Eisenstein - Das Kapital, Berlin, Filmedition Suhrkamp, 2008.

19. - Cf. Alexander Kluge, Sämtliche Kinofilme, dvd n ${ }^{\circ}$ (Deutschland im Herbst, 1978).

20. - Sergueï M. Eisenstein (1898-1948), réalisateur soviétique, auteur de films tels que Le Cuirassé «Potemkine »(1925), Octobre : dix jours qui secouèrent le monde (1928) ou encore Ivan le Terrible (1944).

21. - Tom Tykwer $(* 1965)$, réalisateur allemand, connu auprès du grand public notamment pour des films comme Cours, Lola, cours (Lola rennt, 1998) ou Le Parfum, histoire d'un meurtrier (Das Parfum : Die Geschichte eines Mörders, 2006) d'après le célèbre roman de Patrick Süskind. Sur le rôle de Kluge pour la « relève » du cinéma allemand des années 1980 à nos jours, voir l'essai de Pierre Gras : Good bye Fassbinder ! 
ce film les dix minutes d'une passionnante contribution au sujet du fétichisme de la marchandise selon Marx. Ce qu'il a fait est brillant. Ce n'est pas un choix courant pour un sujet de film. Ainsi le principe de coopération traverse tout le film. Prenez encore la contribution de Werner Schroeter dans ce même ensemble filmique. Dans sa mise en scène de Tristan et Iseult de Richard Wagner, les chanteurs sont habillés comme les marins du Cuirassé Potemkine d'Eisenstein : comme le suggère ce film, l'ardeur révolutionnaire trouve son pendant dans l'ardent amour que thématise l'opéra de Wagner. Cette contribution à son tour se rapporte exactement à la dimension coopérative. Schroeter est mort, hélas, mais je continue à travailler avec lui autant que de son vivant. De même que Heiner Müller reste toujours présent pour moi, bien qu'il soit mort. À mon sens, la permanence des monstres sacrés de la littérature et du cinéma est consubstantielle à la notion d'auteur. Ils constituent l'arsenal des optiques poétiques à côté des optiques traditionnelles dont le cinéma dispose. Sauf que ces dernières n'en sont que les outils techniques. Les vrais instruments, ce sont les yeux des autres.

\section{V.P. : Vos anciens films collectifs avaient la durée normale d'un long- métrage. Vu les proportions de Nouvelles de l'antiquité idéologique, qui s'étend sur près de neuf heures, quels avantages aurait d'après vous le dvd?}

A.K. : Le dvd rend possible une forme qui va de toute manière s'imposer notamment grâce à Internet, une forme qui permet le développement d'ensembles filmiques selon des dimensions conformes au sujet traité. L'année prochaine, nous connaîtrons par exemple les cent ans du déclenchement de la Première Guerre mondiale. À mon avis, on ne peut représenter celle-ci qu'à partir d'une approche prismatique. Il faut croiser les regards qu'on lui porte depuis la France, l'Allemagne, l'Angleterre, les Balkans, Istanbul et Jérusalem. Par exemple, je suis très touché par l'affliction, lors de la nuit de la Saint-Sylvestre 1918, des soldats et des Parisiens pour les quantités de morts dans leur pays et l'étendue des calamités endurées. Les vainqueurs sont en deuil. Au même moment, à Berlin, les vaincus accumulent du ressentiment au fond de leurs cœurs et s'abandonnent à la distraction par des films de divertissement. On s'aperçoit qu'en réalité il n'existe encore aucun récit de la Première Guerre mondiale. Et il est impossible d'y pourvoir en quatre-vingt-dix minutes. Cela est possible en une minute ou en une dizaine, voire une trentaine d'heures, mais pas à l'aide d'une

Le cinéma allemand depuis la réunification, Arles, Jacqueline Chambon (coll. « Rayon Art »), 2011, p. 255-297. 
intrigue de quatre-vingt-dix minutes. C'est en quoi le dvd comporte des avantages décisifs. Pour naviguer sur les eaux de l'expérience, vous pouvez prendre le bateau à vapeur, ou bien vous construisez un radeau. Avec un radeau, vous pouvez descendre le Mississipi, aller du Missouri jusqu'à la Nouvelle Orléans, et même un peu plus loin. Vous pouvez à votre guise agrandir ou réduire les dimensions d'un radeau, principe robuste même s'il n'aboutit pas à une construction aussi parfaite que celle d'un bateau. L'usage du dvd ressemble à cela. C'est un média de transition, qui permet de conserver des données en attendant qu'émane de l'esprit Internet un moyen de concentration similaire. De même qu'il y a dans toute métropole un opéra, un lieu de concentration musicale. Si cela manquait, si chacun dans son immeuble ou à la bourse se contentait de chantonner, l'opéra n'existerait pas.

V.P. : Plus généralement, il semble que dans Nouvelles de l'antiquité idéologique, qui inclut d'ailleurs des passages d'opéras, les moyens artistiques font l'objet d'une méditation beaucoup plus directe et exhaustive que ceci était le cas dans vos œuvres moins récentes. Il y est notamment question d'un projet esquissé par Eisenstein en 1929 de « cinéfier » le Capital de Marx : que nous dit ce projet ?

A.K. : Il est certain qu'on ne saurait porter à l'écran l'ouvrage de Karl Marx, en revanche on peut traduire certains de ses éléments par des scènes si provocatrices qu'elles suscitent une curiosité pour Marx. Par exemple, si vous prenez le concept du caractère fétiche de la marchandise et que vous le restituez par la formule « Tous les objets sont des hommes transformés », qui signifie que chaque objet produit de main d'homme contient une part de travail et de vie humaine - c'est ce en quoi Marx voyait la dimension spirituelle et essentielle du monde de la marchandise -, alors vous pouvez donner une représentation stupéfiante de cette analyse à partir de menus détails. Vous pouvez aussi représenter les réactions humaines par rapport à cela, comme dans cette scène que j'ai consacrée à une idée d'Eisenstein : il y est question des concierges de Paris, ces gardiennes d'immeuble qui existent depuis la Révolution française, qui ont longtemps porté la bannière de la révolution, et sans lesquelles rien ne va dans Paris. Du temps de l'empire du Tsar, celles-ci ont mis toutes leurs économies dans le Transsibérien, et plus tard le gouvernement bolchévique déclarera que ces emprunts russes ne seront pas remboursés. Par la suite aucun parti marxiste ne sortit plus vainqueur d'élections parisiennes, ce qui était dû à l'action des concierges de Paris. Cette histoire n'est pas de moi, mais c'est une idée d'Eisenstein qu'il voulait porter à l'écran. Que ceci lui fût interdit ne m'empêche pas de faire figurer la scène parmi 
l'ensemble de mes films. Je trouve fort intéressante une perspective pareille qui nous en dévoile une seconde, car bien souvent la lecture du Capital de Marx ne s'effectue pas sans difficulté. Il est possible néanmoins de voir à l'œuvre l'écrivain politique brossant le tableau de la guerre de Crimée, la plus exacte description d'une guerre que je connaisse. Dans ces rapports quotidiens rédigés pour un journal américain, il retrace comment le régime du Tsar émet une obligation qui sera vendue par des banquiers belges, français et britanniques pour financer les obus russes qui seront d'abord dirigés contre l'expédition armée de Crimée menée par des Français, des Italiens, des Turcs et des Anglais, ce qui constitue des liens extrêmement intéressants et une métaphore permettant de mieux comprendre le caractère abstrait des flux de marchandises et d'argent (la mondialisation financière, alors que la guerre reste locale).

\section{V.P. : En somme, la question est de savoir comment changer en images des notions philosophiques parfois arides, voire poussiéreuses.}

A.K. : Ceci est faisable. Du moins est-ce l'exigence qu'en tant que fouilleurs, archéologues de la littérature, du cinéma et de l'histoire nous devons avoir aujourd'hui. La modernité s'est accomplie depuis longtemps. Bien des choses sont déjà relatées, mais sans être suffisamment mises en relation. Cette qualité de l'archéologue, que tout poète conscient de lui-même devrait avoir de nos jours, peut s'exercer en déterrant, transcrivant, transformant, exposant et corrélant.

V.P. : Un peu comme vous le faites lorsqu'au départ de l'actuelle crise financière vous entamez une coopération virtuelle avec le Sergueï Eisenstein de 1929 et du début de la crise des années 1930 afin de faire revivre l'œuvre de Karl Marx : en ce sens, n'y aurait-il pas lieu d'interpréter Nouvelles de l'antiquité idéologique comme une proposition contre la théorie de Fukuyama?

A.K. : En fait, je ne pensais pas à Fukuyama que je situe assez loin de mes préoccupations. D'ailleurs, je ne fais pas d'ouvrages à thèse, c'est-à-dire des livres de combat, car je n'écris pas sur le mode discursif. Mais ce procédé qui consiste à s'attacher par induction au détail, de manière à permettre ensuite la compréhension de l'ensemble, et dont les résultats ne conduisent pas non plus aux thèses de Fukuyama, représente quelque chose d'essentiel, une méthode qu'à mon tour je tiens de Montaigne, lequel ne part jamais d'une conception générale afin de déterminer d'après une théorie globale où est le bien, où le mal, mais ne retient l'attention qu'à l'aide d'une approche inductive et détaillée, nourrie toutefois par l'intégralité des sources antiques. En tant que station-relais de l'Antiquité, Montaigne est une OASIS 
DE L'ATTENTION. Et c'est ce dont Internet a besoin aujourd'hui : des centres de gravité, des champs de force, des champs gravitationnels de l'attention. Au fond, c'est cela que nous faisons, sans qu'il nous faille échafauder des thèses que les hommes conçoivent d'eux-mêmes. Si maintenant vous placez Fukuyama dans ce laboratoire d'alchimie et le plongez, pour ainsi dire, dans un bain d'acide, vous verrez que c'est là tout sauf de l'or.

V.P. : Puisque vous revenez aux Essais de Montaigne, Eisenstein affirmait lui aussi ne pouvoir se passer des notions génériques propres à la littérature pour les appliquer au septième art encore jeune à l'époque : en l'occurrence, son approche doit beaucoup à Joyce 22 . Comment voyez-vous aujourd'hui le rapport entre ces deux arts ?

A.K. : Il demeure complètement inchangé. Si Joyce et Eisenstein se rencontrent à Paris en 1929 durant la même semaine que celle du Jeudi noir, pour imaginer ensemble comment faire un film sur le Capital de Marx, c'est que leur rapport est très proche. Un James Joyce quasiment aveugle, peu à même sans doute de voir les images et les esquisses que lui présente Eisenstein, donne à ce dernier le courage d'opter pour une forme de narration libre et non linéaire. Voici donc que la littérature enrichit considérablement le cinéma, lequel demeure quant à lui un moyen de traduire par des scènes ce que Joyce ne pourrait évoquer que de façon cryptée par le verbe. La combinaison des programmatiques de Joyce et d'Eisenstein garderait en 2014 toute l'actualité et la modernité qui fut déjà la sienne en 1929.

V.P. : Dans votre film, quel est le rôle qui revient à la figure d'Ovide, qui résume et assemble toutes les métamorphoses dans son chefd'œuvre du même nom ? Par quel chemin la tisseuse Arachné rejoint-elle la toile de cinéma ?

A.K. : Arachné tissant sa toile est pour moi le symbole du réseau Internet. Cette femme fabrique des habits (des textes) où elle dessine toute l'histoire du monde. Si je recouvre ma peau de quelque chose comme d'un vêtement, cela me fait comme une seconde peau. Et sur cette seconde peau elle déploie des micro-récits. Elle l'emporte sur la déesse Athéna qui tente de faire de même, mais compose avec des principes au lieu de raconter. Ainsi, cette tisseuse supplante Athéna. Pour la punir, la déesse la transforme en araignée. L'Arachné d'Ovide est en quelque sorte la déesse patronne d'Internet, des rapports pré-

22. - Voir l'article de Tobias V. Powald, «L'antique, l'authentique : Alexander Kluge ou les métamorphoses d'Hermès », in : Germanica, $\mathrm{n}^{\circ} 45$, Université Charles de Gaulle - Lille III, 2009, p. 125-141. L'article est désormais disponible en ligne : http:// germanica.revues.org/832. 
sents dans un film ou à l'intérieur d'Internet, en supposant que ces rapports existent dans ce réseau-là. En tout cas, il doit bien exister un Dieu capable de voir et de déchiffrer ces relations. En tant qu'individus, nous ne le pourrions pas, car tout cela nous dépasse un peu. Cependant, il nous reste la représentation, c'est-à-dire le prisme. Pour revenir à l'une des questions précédentes : où sur le Net se trouve ce lieu que l'opéra occupe dans une grande ville ? Si je m'exprime par des métaphores lorsque je dis qu'il convient de creuser des puits, créer des oasis quand il y a trop de silice, trop de désert alentour, et que lorsqu'on ne peut nager en mer, d'une rive à l'autre, entre l'Irlande et les États-Unis - la distance étant un peu trop longue pour un simple nageur -, nous devons prendre un véhicule, que ce soit un bateau, un radeau, voire un dirigeable ou encore un sous-marin, bref, il faut comprendre que les formes ainsi figurées sont à peine en train d'émerger. Cela dit, il me semble que les voies de l'esprit développent des effets gravitationnels et finissent par être découvertes, mais seulement au bout d'un certain temps, tel un message dans sa bouteille. La qualité requise pour ce genre d'oasis sur le Web défie en réalité la poétique. J'imagine Honoré de Balzac, qui fut un entrepreneur, travaillant avec nous aujourd'hui. Il dirait sûrement que nous devons éviter d'ajouter de nouveaux ensembles romanesques aux nombreux romans qui existent déjà, et défendrait l'idée du ROMAN SUR INTERNET. Ces concentrés n'appartiendraient à personne. Le complément approprié d'Edward Snowden serait un Balzac tissant de la narration sur Internet.

V.P. : Dans votre film Nouvelles de l'antiquité idéologique on a également beaucoup affaire à des écrits, textes lus à haute voix par les acteurs, textes à lire par les spectateurs, textes chantés, récités, discutés et expliqués. En quoi est-ce « filmique » et quel est l'effet escompté ?

A.K. : Tout d'abord, j'ai repris ce procédé du cinéma muet, car celui-ci connaissait les intertitres. Dans le Docteur Mabuse de Fritz Lang23, l'intrigue est exclusivement véhiculée par les intertitres, donnant libre cours à l'improvisation filmique d'un intertitre à l'autre, pour les paraphraser, à la création de scènes qui ne soient pas encombrées de dialogue informel. Cela peut s'écarter considérablement du théâtre,

23. - Fritz Lang (1890-1976), réalisateur du célèbre Metropolis (1927), est d'une certaine manière à l'origine de la carrière cinématographique d'Alexander Kluge qui, sur la recommandation de Theodor W. Adorno, assista au tournage du diptyque composé du Tigre du Bengale (Der Tiger von Eschnapur, 1958) et du Tombeau hindou (Das indische Grabmal, 1959). Docteur Mabuse le joueur (Doktor Mabuse, der Spieler), que Kluge évoque ici, date de l'année 1922. 
étant donné que le texte écrit domine. Et j'emprunte ce moyen-là au cinéma muet, car il me paraît évident qu'un mot écrit contient une image au même titre qu'une illustration. Si nous avons affaire de nos jours à un surcroît d'images, notamment parce que la force de persuasion publicitaire vient s'ajouter à toutes les images qui, en quête de succès, défilent à la télévision, ces images-là sont aujourd'hui, pour ainsi dire, usées jusqu'à la corde. Dans un environnement pareil, je suis iconoclaste. Les bons films ont d'ailleurs la propriété de détruire des images : ils remplacent les mauvaises images par des images rares et bonnes.

V.P. : Il n'est pas rare de voir dans votre œuvre cinématographique d'importants passages scripturaux, voire des récits entiers qui demandent à être lus à l'écran, et où vous assumez très radicalement cette complémentarité des effets du cinéma et de la littérature. Pourquoi cette forme?

A.K. : C'est tout simplement la seule solution, dès lors qu'il m'est difficile de convaincre par l'image. Quand je dois préparer une image, ou encore en isoler une par rapport à d'autres, je peux le faire en alternant avec des séquences écrites qui demandent à être lues et à ce qu'on se fasse son idée. Au demeurant, cela n'est pas uniquement une affaire d'écriture, puisque celle-ci y est accompagnée de musique et émaillée d'images. On mesure l'intensité avec laquelle ces images insérées dans la représentation écrite restent en mémoire. Nous pourrions maintenant faire le test, seulement, si j'observe cette démarche, ce n'est pas pour des raisons scientifiques, mais narratives, parce que je suis absolument certain qu'il s'agit-là d'une forme narrative moderne et nécessaire.

V.P. : De telles œuvres ne montrent-elles pas combien le montage, aussi bien comme moyen essentiel du cinéma qu'en tant que procédé littéraire, si l'on pense notamment au mouvement Dada, puisse leur tenir lieu de dénominateur commun?

A.K. : Notez qu'avec Christoph Marthaler et d'autres, nous avons produit un film d'environ six heures sur Dada. Et vous dites vrai, car en réalité Dada fait partie du cinéma. En fait, la distinction entre le cinéma et le mot n'existe pas dans les arts du $\mathrm{Xx}^{\mathrm{e}}$ siècle, car la littérature du $\mathrm{XX}^{\mathrm{e}}$ et $\mathrm{XXI}^{\mathrm{e}}$ siècle n'est pas tout à fait identique à celle qui a précédé. Ulysse de Joyce renvoie certes à Homère et à l'Antiquité, mais en même temps, il s'agit-là d'une chose entièrement inédite et innovante qu'Homère n'aurait jamais faite, et de ce point de vue l'Avant-garde des années 1920 et 1930 constitue en fait un héritage que nous ne continuons pas en le niant, mais en le stabilisant et en retournant à 
ses racines. Nous creusons nos puits dans le champ de la modernité, pour nous rendre compte avec étonnement que la modernité n'a rien de nouveau, que l'idée existait déjà dans l'Antiquité, bien avant d'être synthétisée en tant que telle. Et voici qu'au XXI ${ }^{\mathrm{e}}$ siècle, une situation urgente se présente, du fait que sept milliards d'humains vivent désormais sur terre, dont un grand nombre participe aux réseaux, et que d'avides multinationales sont à même de rassembler ces masses humaines comme aux temps des Congrès du Reich, de manière à réunir un même soir pour une heure cette foule composée de millions de spectateurs autour des structures télévisuelles : une autoroute de l'esprit pas si facile à traverser pour une simple tortue. Où même des renards se font happer. Quoi que vous fassiez en tant qu'individu ou auteur, vous demeurez d'abord impuissant face au déferlement organisé de cette masse immense, sans le moindre pouvoir contre ce Congrès du Reich du divertissement.

\section{V.P. : En quoi justement le recours au montage s'avère-t-il décisif pour un auteur dans pareil contexte?}

A.K. : Nous savons nous servir du montage, contrairement à eux. C'est nous qui sommes en possession des moyens artistiques. Le montage importe bien davantage que l'image proprement dite. Ainsi vous pouvez monter des édifices de pensée ou des ensembles émotionnels. La trame est extrêmement riche en réalité, ce qui se traduit par sa dimension prismatique et d'autant mieux que vous saisirez l'effet de montage comme une forme de la richesse, de la diversité, de la polyphonie des images, des significations, des pauses entre les significations, de la restitution de l'autonomie à des objets isolés même dépourvus de signification, comme la capacité, en quelque sorte, de générer du sens et de pallier la FAIM DE SENS, mais aussi de développer par ailleurs l'autonomie et la LIBERTÉ DE TOUTE CONTRAINTE DE SENS. Ces moyens très subtils et très fins, jamais grossiers, forment tous ensemble une richesse.

\section{V.P. : Comment distingueriez-vous le montage de la citation, celle-ci étant plus couramment associée à la littérature ?}

A.K. : La citation est l'un des procédés du montage. Elle réfère toujours à quelque chose et ne demeure jamais sans contexte. Car il ne s'agit pas de reprendre quelque chose, mais de l'extraire pour l'insérer dans un nouveau contexte, où cela se transforme.

V.P. : Qualifieriez-vous Nouvelles de l'antiquité idéologique de film de montage ou plutôt de film-essai, pour en revenir à une catégorie littéraire par son origine? 
A.K. : Sans doute en jugeriez-vous mieux que moi-même, qui ne suis pas critique de profession. J'y verrais tout aussi bien une chronique. Peut-être n'est-ce pas un essai, vu les nombreuses scènes prises sur le vif qui s'y trouvent intégrées. Aussi, l'argumentation ne s'y effectue pas sur le mode de l'essai. Mais je ne contesterais pas non plus la désignation d' « essai ». Si vous l'entendez au sens de Montaigne, vous pouvez l'utiliser sans problème.

V.P. : En littérature comme au cinéma, le montage est censé, selon vous, produire des contrastes, susciter même l'épiphanie, afin que l'art de distinguer opère. Pourriez-vous expliciter cette idée ?

A.K. : Je crois que tout ce dont nous soyons capables en tant qu'auteurs, qu'il soit question de cinéma, de musique ou de littérature, tend à stabiliser et à aiguiser la capacité de distinguer, c'est-à-dire à collectionner les différences. L'épiphanie quant à elle consiste à faire émerger au travers d'objets une seconde image intérieure qui renvoie à quelque chose d'essentiel. Chez Theodor W. Adorno et Walter Benjamin, l'équivalent du concept théologique de l'épiphanie est celui de l' « image dialectique ». La capacité de distinguer spécifique à l'œuvre ici se joue entre l'image extérieure visible et une image intérieure, l'épiphanie justement.

V.P. : En fin de compte, l'enjeu est de recueillir du matériau destiné à stimuler l'imagination du lecteur ou du spectateur, à rendre possible l'expérience intérieure, ce qu'Eisenstein entendait faire lorsqu'il trouva dans le monologue intérieur joycien un moyen de traduire la pensée conceptuelle en pensée émotionnelle et sensuelle...

A.K. : Ces trois choses se font en même temps, comme si vous superposiez trois cartes, de manière à obtenir une sorte de configuration, un champ contextuel ou narratif. Cet espace narratif ne s'ouvre jamais de façon unidimensionnelle, seulement par des lettres, des images, des concepts, des émotions ou par du sensuel à tout va. Tous ces niveaux se parlent entre eux, au même titre que les notes d'un air de musique polyphonique luttent, créent des tensions, communiquent : le tout forme la trame narrative.

V.P. : Mais si la littérature et le cinéma naissent « dans la tête » du récepteur, comme il vous arrive d'affirmer, ceci ne signifie-t-il pas que cela repose justement sur des expériences élémentaires plus que sur les techniques qui les encouragent ?

A.K. : Les différences sont d'ordre élémentaire en même temps qu'elles définissent un rapport. L'un n'exclut pas l'autre. Partons du principe qu'en tant qu'animaux à sang chaud, nous les êtres humains avons 
d'abord appris de nos ancêtres cette distinction élémentaire entre le chaud et le froid. Cela est vital pour nous, parce qu'il ne doit faire ni trop chaud ni trop froid, qu'il nous faut trouver un milieu de vie, etc., ce dont nos ancêtres se sont acquittés avec beaucoup d'habileté. Sur cette distinction élémentaire entre chaud et froid reposent toutes les distinctions plus complexes : proche/éloigné, vénéneux/bénéfique, bon/méchant. Les distinctions plus complexes requièrent un niveau de discernement assez élevé. Il est nécessaire de les ramener en permanence à leur dimension élémentaire. Les distinctions morales à leur tour se fondent sur le simple fait que la majorité de l'espèce humaine n'a pu survivre à l'évolution qu'en raison de sa bonté naturelle, autrement dit grâce à la coopération sociale. Ces qualités sont particulièrement fiables, ce qui ne vaut pas forcément pour les préceptes moraux. Et faire la part entre toutes ces choses-là me paraît plus facile en français que dans ma propre langue, car les langues latines sont attachées aux différences. Un livre de Claude Lévi-Strauss est pour ainsi dire une compilation de cette aptitude à distinguer. En ce sens, les films de Jean-Luc Godard sont eux aussi des compilations du discernement visuel, sensuel et mental.

V.P. : En son temps, Robert Musil était lui aussi très conscient de l'impact possible de l'expérience surtout visuelle du cinéma de son époque, dont cependant il attribuait la signification avant tout à l'effet symbolique des images ${ }^{24}$. Partageriez-vous cette opinion ?

A.K. : Pour commencer, j'hésiterais, puisque l'effet symbolique des images ne crée pas d'images nouvelles et gêne plutôt le discernement. Il vaudrait mieux en tous cas regarder un film exempt de substance symbolique, donc dépourvu de sens : un film burlesque, par exemple, se regarde sans qu'il faille être attentif au sens. Dans les films d'Ernst Lubitsch, la narration ne procède souvent d'aucune contrainte liée au sens. Ceci m'intéresse davantage que de le voir s'engager dans un film instructif tel que Jeux dangereux ${ }^{25}$, dont l'intrigue renvoie symboliquement à Hitler et dont les images deviennent très vite lourdes de signification.

\section{V.P. : L'intrigue de L'Homme sans qualités de Robert Musil nous fait remonter à l'année 1913, où l'art cinématographique était encore à ses débuts qu'on qualifiera de primitive diversity, à laquelle vous vous}

24. - Voir l'essai de Robert Musil, «Remarques sur la dramaturgie du cinéma » (1925), in : Daniel Banda/ José Moure (Éds.) : Le cinéma : l'art d'une civilisation, Paris, Flammarion/Champs (coll. « arts »), 2011, p. 94-98.

25. - Jeux dangereux (To be or not to be) d'Ernst Lubitsch (1892-1947) est sorti dans les salles en 1942. 
référez. En quoi est-elle d'actualité cent ans plus tard et comment cette forme populaire s'accomplit-elle de nos jours?

A.K. : La notion de primitive diversity renvoie à la forme ancestrale du cinéma, avant que d'astucieux producteurs ne se mirent à lui imposer le modèle théâtral, prévoyant ainsi des drames en trois actes, du divertissement et des effets. De par sa tendance à l'expérimentation, le cinéma de la première heure présente quelque chose de passionnant : la simplicité dans la diversité. De la sorte paraissent des images que vous n'avez jamais vues, ce qui est très rare au cinéma, car pour un film hollywoodien normal ou tout autre film d'usage, il vous faut quantité d'images qui, grâce à leur sens et leur substance symbolique, peuvent se comprendre et se décrypter immédiatement. Un film de cette espèce ressemble à de l'espéranto. Mais un vrai film fait se succéder les surprises, car tout ce que la caméra peut enregistrer spontanément, sans que l'entendement humain ne s'immisce, a pour vertu de surprendre. Si vous filmez un fourré, soigneusement, avec les acariens et tout ce qu'on pourrait y voir, vous auriez-là un univers inconnu et vous vous retrouveriez comme sur une autre planète. Le cinéma est un moyen radical qui crée de lui-même une distance, cette distanciation allant de pair avec l'exploration. Par là-même on s'aperçoit que les rapports réels diffèrent des significations qu'on leur donne.

V.P. : Dans l'une de vos histoires qui traite d'un projet d'adaptation de L'Homme sans qualités, l'aspect d'un « Film sans qualité » est discuté, en référence au célèbre roman « sans qualités » tout empreint d'essayisme qu'est celui de Robert Musil. Dans quelle mesure cette solution vous paraît-elle adéquate ?

A.K. : Robert Musil concentre toutes ces formes. Tout ce que nous venons d'évoquer, nous le trouverions chez Musil. Si j'avais maintenant la mission de décrire ce $\mathrm{XXI}^{\mathrm{e}}$ siècle avec le regard de Musil, c'est-à-dire dans la perspective d'une journée du mois d'août 1913, je partirais de la seconde partie inachevée de L'Homme sans qualités. Résidant à Genève d'où il observe les événements de l'année 1941 en Europe, Musil lui-même le suggérait à la fin de son immense ouvrage, lorsqu'il déclare dans un fragment d'une dizaine de lignes qu'Ulrich devrait décrire la Seconde Guerre mondiale avec le regard de 1913.

\section{V.P. : De quelle manière faudrait-il s'y prendre, afin d'adapter ce roman au cinéma?}

A.K. : Je le découperais en fragments, puisqu'il est de toute façon un fragment et qu'il m'importe justement de prendre Musil au sérieux. Mieux vaut porter à l'écran un simple paragraphe que l'intégralité 
du roman, lequel ne procède d'aucune intrigue dont vous puissiez tirer un scénario. Plutôt que dans la relation d'Ulrich avec sa sœur, l'essentiel réside dans cette journée d'été où il pleuvait des fleurs et qui reste gravée dans leur mémoire. Le vrai sujet est celui-là, et non les rapports incestueux entre le frère et la sœur. En revanche, cette scène me séduit notamment pour deux raisons très différentes, la première étant qu'on y voit l'auteur brosser le tableau d'une journée d'été avec une maîtrise qui, ma foi, n'aurait rien à envier à celle de Gerhard Richter ${ }^{26}$. La seconde est le reflet qu'elle donne de toutes les journées d'été d'avant-guerre. Pour moi, tout grand jour d'été magnifiquement ensoleillé n'a rien d'un sujet pour un poème de Rilke, mais témoigne de l'inquiétude avec laquelle on s'attendait qu'à l'issue de l'été 1914 la guerre vienne à éclater. J'ai vécu la même chose en 1939 quand j'étais enfant. Et je viens de constater qu'à la période la plus dangereuse de la Guerre froide, l'été souriait à l'heureux père d'une petite fille âgée de cinq mois que j'étais, alors que je présentais un film au Festival de Venise. C'était la belle saison, et me voilà installé avec mon épouse et cet enfant dans une cabane de plage du Lido, heureux, me laissant prendre en photo par un photographe des Cahiers du Cinéma. En 2013, j'apprends qu'à ce moment idyllique nous avons vécu la semaine la plus tendue de la Guerre froide, durant laquelle un funeste malentendu s'était produit entre les Russes (Andropov) et les Américains (Reagan). À la différence de la situation actuelle en Syrie, l'un des deux camps n'avait même pas réalisé ce qui affolait l'autre. Sur les écrans de contrôle détraqués de la défense anti-missile, on avait observé des signaux qui semblaient indiquer que des missiles traversaient le Pôle Nord pour une frappe destinée à décapiter le commandement russe. La suite allait montrer qu'on faisait erreur. Mais il s'en est fallu de peu qu'en cette fin d'été de 1983 on passe d'une Guerre froide à une Guerre chaude. Les beaux jours et le danger suprême, l'idylle et l'abîme sont proches, et c'est ainsi qu'à travers l'expérience de ce siècle la scène estivale extraite de l'œuvre de Musil se mue en une perspective qui n'a plus rien à voir avec le moment décrit par lui. Dans le même temps son roman, qui traite de la FORME DU POSSIBLE, offre carrément l'incitation et le cadre qui permettent d'associer de tels éléments.

\section{V.P. : Comment faut-il interpréter le malentendu qui oppose l'auteur- réalisateur et le producteur dans le dialogue de votre texte L'Homme sans qualités, qui emprunte son titre à Musil ?}

26. - Au sujet des œuvres co-réalisées par Alexander Kluge et Gerhard Richter, voir la note 1 . 
A.K. : D'une certaine manière, il s'agit-là d'une histoire autobiographique, dont j'ai juste un peu forcé le trait. Une attitude comme celle de ce jeune réalisateur signifie à mes yeux la possibilité de faire des films audacieux, modernes, neufs, surprenants. Il est vrai aussi qu'ils ne risquent pas de s'adresser à une majorité, étant donné que la propagande des diffuseurs et les habitudes prises vont dans l'autre sens. Raison pour laquelle à la télévision nous tendons des « pièges » à ceux qui zappent. Comme à la télévision le samedi soir tout semble assez uniforme, les spectateurs zappent, voient cette uniformité en y cherchant ce qui les intéresse le plus. S'il passe alors quelque chose d'inconnu, où rien de ce qui se passe généralement à la télévision ne se produit, ils se retrouvent soudain à écouter deux personnes, deux Directeurs d'Institut Max Planck d'astrophysique, qui se racontent des choses à propos de trous noirs ou de galaxies se situant à trois cents années-lumière à peine du commencement de l'univers. Comme ils y vont de leur façon de synthétiser, avec le langage scientifique qu'ils se sont forgés, cela paraît parfaitement abscons au premier abord, mais vous serez étonné de voir qu'en piégeant les zappeurs vous êtes en mesure de réunir toute une foule de jeunes gens, c'està-dire une majorité momentanée et véritable au sein du groupe-cible. Ils voient quelque chose d'inconnu qui les intéresse davantage que la somme de tout ce qui est connu et attrayant. C'est en quoi le poète dispose d'un allié en chaque individu conscient de soi.

Traduction : Vincent PAUVAL 